\title{
In vitro cell behaviors of bone mesenchymal stem cells derived from normal and postmenopausal osteoporotic rats
}

\author{
QIAN LIU ${ }^{1 *}$, XIAOXIA ZHANG ${ }^{2 *}$, YANG JIAO $^{3 *}$, XIN LIU $^{4}$, YIRONG WANG ${ }^{4}$, SONG-LUN LI $^{5}$, \\ WEI ZHANG ${ }^{6}$, FA-MING CHEN ${ }^{7}$, YIN DING ${ }^{1}$, CHUAN JIANG $^{8}$ and ZUOLIN JIN ${ }^{1}$
}

${ }^{1}$ State Key Laboratory of Military Stomatology and National Clinical Research Center for Oral Diseases and Shaanxi Clinical Research Center for Oral Diseases, Department of Orthodontics, School of Stomatology, The Fourth Military Medical University, Xi'an, Shaanxi 710032; ${ }^{2}$ Department of Stomatology, Xi'an Medical College, Xi'an,

Shaanxi 710309; ${ }^{3}$ Department of Stomatology, PLA Army General Hospital, Beijing 100700; ${ }^{4}$ State Key Laboratory of Military Stomatology, Department of Operative Dentistry and Endodontics, School of Stomatology,

The Fourth Military Medical University; ${ }^{5}$ Medical Service Management Office, Tangdu Hospital,

The Fourth Military Medical University; ${ }^{6}$ Research Center of Traditional Chinese Medicine, Xijing Hospital,

The Fourth Military Medical University; ${ }^{7}$ State Key Laboratory of Military Stomatology,

Department of Periodontology, School of Stomatology, The Fourth Military Medical University, Xi'an, Shaanxi 710032; ${ }^{8}$ Department of Stomatology, The People's Hospital of Tongchuan, Tongchuan 727000, P.R. China

Received March 22, 2016; Accepted October 19, 2017

DOI: $10.3892 /$ ijmm.2017.3280

\begin{abstract}
Postmenopausal osteoporosis (PMO) increases bone fragility and the risk of fractures, and impairs the healing procedure of bone defects in aged women. The stromal cell-derived factor- $1 \alpha(\mathrm{SDF}-1 \alpha) / \mathrm{CXC}$ chemokine receptor type 4 (CXCR4) axis helps to maintain the biological and physiological functions of bone marrow mesenchymal stem cells (BMSCs) and increase the homing efficiency of BMSCs. The present study aimed to
\end{abstract}

Correspondence to: Dr Chuan Jiang, Department of Stomatology, the People's Hospital of Tongchuan, 12 Jiankang Road, Tongchuan 727000, P.R. China

E-mail: jiangchuanfei@163.com

Professor Zuolin Jin, State Key Laboratory of Military Stomatology and National Clinical Research Center for Oral Diseases and Shaanxi Clinical Research Center for Oral Diseases, Department of Orthodontics, School of Stomatology, the Fourth Military Medical University, 145 Changle West Road, Xi'an, Shaanxi 710032, P.R. China E-mail: zuolinj@163.com

*Contributed equally

Abbreviations: BMSCs, bone marrow mesenchymal stem cells; PMO, postmenopausal osteoporosis; SDF-1, stromal cell-derived factor-1; CXCR4, CXC chemokine receptor type 4; PBS, phosphatebuffered saline; $\alpha$-MEM, $\alpha$-minimum essential medium; FBS, fetal bovine serum; micro-CT, micro-computed tomography; CFUs, colony-forming units

Key words: postmenopausal osteoporosis, bone marrow mesenchymal stem cells, stromal cell-derived factor- $1 \alpha / \mathrm{CXC}$ chemokine receptor type 4 , cell homing, migration ability, osteogenic ability provide insights into the possible association between migration and osteogenic ability and the SDF-1 $\alpha /$ CXCR4 axis in BMSCs derived from a rat model of PMO. In order to do this, the general and SDF-1 $\alpha /$ CXCR4-associated biological characteristics as well as associated molecular mechanisms in BMSCs isolated from a PMO rat model (OVX-BMSCs) and normal rats (Sham-BMSCs) were investigated and compared. In comparison with ShamBMSCs, OVX-BMSCs exhibited an impaired osteogenic ability, but a stronger adipogenic activity as well as a higher proliferative ability. In addition, OVX-BMSCs presented a lower chemotactic activity towards SDF-1 $\alpha$, lower expression levels of CXCR4 and reduced levels of phosphorylated AKT (p-AKT). Therefore, the lower expression levels of CXCR4 and p-AKT may be responsible for the impaired osteogenic ability and lower chemotactic activity towards SDF-1 $\alpha$ of OVX-BMSCs.

\section{Introduction}

Postmenopausal osteoporosis (PMO) is a worldwide health problem $(1,2)$. It is a systemic skeletal disease characterized by a reduction of bone density and micro-architectural deterioration, which increases the risk of fractures in aged women $(3,4)$. Estrogen serves a principal role in the processes of skeletal growth and bone homeostasis as well as bone metabolism (5). However, in postmenopausal women, the deficiency of estrogen results in a reduction of bone mass (6). Experimental evidence suggests that in osteoporotic patients, osteoblast activity reduces whereas osteoclast activity increases, and thus bone resorption exceeds bone formation (4,7). Therefore, the focus of cytological experiments on the origin of PMO has been osteoblast activity and bone resorption processes, followed by osteoblast genesis and the differentiation potential of bone marrow mesenchymal stem cells (BMSCs) (8). BMSCs are pluripotent and capable of self-renewal, supporting hematopoiesis, bone marrow formation 
and differentiating into multiple tissues in vitro and in vivo $(9,10)$. Studies have demonstrated that BMSCs derived from osteoporotic postmenopausal women exhibit disturbed intrinsic properties and impaired differentiation ability compared with normal BMSCs (11). However, it is unclear whether the BMSCs from a rat model of PMO are similarly affected.

Stromal cell-derived factor- $1 \alpha$ (SDF- $1 \alpha)$ and its cognate receptor CXC chemokine receptor type 4 (CXCR4) have been reported to be highly active in the reparation and regeneration of various injured tissues by promoting the migration of stem cells $(12,13)$. In addition, SDF-1 $\alpha$ is considered by various researchers as a major homing factor for the targeting of hematopoietic stem cells and mesenchymal stem cells (MSCs), both of which express CXCR4, to the bone marrow (14-16). Recent studies suggest that the CXCR4/SDF-1 $\alpha$ axis may be important in maintaining the biological and physiological functions of BMSCs $(17,18)$. Furthermore, the axis may help to increase the homing efficiency of BMSCs (19). Increasing the concentration of SDF-1 $\alpha$ has been demonstrated to cause a dose-dependent induction of the chemotactic effects of MSCs $(20,21)$. Other studies have identified an association between decreased CXCR4 expression in BMSCs and endothelial progenitor cells and their functions in tissue regeneration $(22,23)$.

On the basis of these observations, it is hypothesized that: i) CXCR4 expression in BMSCs isolated from a PMO rat model (OVX-BMSCs) is reduced; and ii) CXCR4-deficiency affects the migration ability and osteogenic differentiation of OVX-BMSCs. To evaluate these hypotheses, the general and CXCR4-related biological characteristics of OVX-BMSCs and normal rat (Sham-BMSCs) were examined and compared using in vitro experiments in the present study. In addition, the chemotaxis of the BMSCs towards SDF-1 $\alpha$ and associated molecular mechanisms were investigated. To the best of our knowledge, this is the first study to conduct a comparison of Sham-BMSCs and OVX-BMSCs using a PMO rat model.

\section{Materials and methods}

Experimental animals and ethics statement. A total of 60 female Sprague-Dawley (SD) rats aged 8 weeks and weighing $200 \pm 20 \mathrm{~g}$ were purchased from the Laboratory Animal Center of the Fourth Military Medical University (Xi'an, China). All protocols were approved by the Institutional Animal Care and Use Committee at the Fourth Military Medical University and were consistent with the Guidelines of Intramural Animal Use and Care Committee of the Fourth Military Medical University. The animals had free access to food and water.

Establishment of the animal models. According to the techniques established by Wronski et al (24), the rats underwent ovariectomy $(\mathrm{OVX}, \mathrm{n}=30$ ) or a sham surgery (Sham, $\mathrm{n}=30)$, following the intraperitoneal injection of $2 \%$ pentobarbital sodium (Nembutal; Abbott Laboratories, Lake Bluff, IL, USA) at a dose of $30 \mathrm{mg} / \mathrm{kg}$. Following surgery, the rats were raised with a 12/12-h light/dark cycle in a temperature-controlled $\left(21-23^{\circ} \mathrm{C}\right)$ room with a relative humidity of $50-60 \%$. All the rats from each group were sacrificed at 3 months following the surgery. To verify the animal model, micro-computed tomography (micro CT; Siemens Inveon Micro-CT; Siemens AG, Munich, Germany) was used to observe the femoral bone mass changes in the OVX and Sham groups. All femoral bone samples were totally scanned and 3D images were constructed. The CT settings were as follows: Voltage, $40 \mathrm{kV}$; current, $250 \mu \mathrm{A}$; voxel size, 10x10 $\mu \mathrm{m}$; slice thickness, $20 \mu \mathrm{m}$; exposure time, 3,000 msec; pixel matrix, 1,024x1,024. The bone mineral content (BMC), bone mineral density (BMD) and ratio of bone total volume/trabecular volume (BV/TV) were evaluated using analytical software (IRW 3.0; Inveon Research Workplace; Siemens AG). The Max load was tested using a universal mechanical testing machine (AGS 10 kN; Shimadzu Corporation, Kyoto, Japan). Osteoporosis in the rats was defined as a BMD of $>2.5$ standard deviations (SDs) below the mean value acquired from the sham group as previously described (25).

Isolation and culture of BMSCs. Rats BMSCs from the Sham and OVX groups were isolated and cultured according to previously reported methods with minor modifications (26). Briefly, tibias and femurs were removed after the rats were euthanized. After excision of the epiphysis, bone marrow from bilateral rat tibias or femoral diaphyses was flushed with $\alpha$-minimum essential medium ( $\alpha$-MEM; Gibco; Thermo Fisher Scientific, Inc., Waltham, MA, USA) supplemented with $10 \%$ fetal bovine serum (FBS; Hangzhou Sijiqing Biological Engineering Materials Co., Ltd., Hangzhou, China) and $1 \%$ penicillin and streptomycin, and incubated at $37^{\circ} \mathrm{C}$ in a humidified atmosphere of $5 \% \mathrm{CO}_{2}$. The culture medium was changed every 2-3 days. When the cells approached $\sim 80 \%$ confluence, adherent cells were treated with a $0.25 \%$ trypsin solution and passaged. Cells at passages (P) 2-6 were used in subsequent experiments. The morphology of the cultured cells was observed and documented by phase contrast microscopy (CKX41; Olympus Corporation, Tokyo, Japan).

Colony formation assay. BMSCs (P3) were plated into 9-cm-diameter culture dishes (Costar) at a density of $1 \times 10^{3}$ cells/dish and cultured in $\alpha$-minimum essential medium ( $\alpha$-MEM; Gibco; Thermo Fisher Scientific, Inc.) supplemented with $10 \%$ fetal bovine serum (FBS; Hangzhou Sijiqing Biological Engineering Materials Co., Ltd.) and $1 \%$ penicillin and streptomycin (Gibco) prior to the colony formation assay. The medium was changed every 2 days, and after 14 days, the cells were fixed in $4 \%$ paraformaldehyde at $4^{\circ} \mathrm{C}$ for $30 \mathrm{~min}$ prior to staining with $0.1 \%$ crystal violet $25^{\circ} \mathrm{C}$ (Sigma-Aldrich; Merck $\mathrm{KGaA}$, Darmstadt, Germany) for 10-15 min, and observation under a stereomicroscope. The number of colony-forming units (CFUs; $\geq 50$ cells) was quantified for statistical analysis.

Cell proliferation assay. BMSCs (P3) were plated into 96-well dishes (Costar) at a density of $2 \times 10^{3}$ cells/well and cultured in complete medium for 1-10 days and the medium was refreshed every 3 days. According to the instructions of the Cell Counting kit-8 (CCK-8; Beyotime Institute of Biotechnology, Shanghai, China), CCK-8 solution was added to each well at a fixed time of day. Following incubation for $4 \mathrm{~h}$ at $37^{\circ} \mathrm{C}$ in the dark, the absorbance of the colored solution was measured using a microplate reader (Bio-Rad Laboratories, Inc., Hercules, CA, USA) at a wavelength of $450 \mathrm{~nm}$.

Flow cytometric analysis. The cells were blocked for $1 \mathrm{~h}$ in PBS containing 5\% (w/v) non-fat dry milk and incubated at $4^{\circ} \mathrm{C}$ overnight. The immunophenotype of the cultured BMSCs (P3) was analyzed using flow cytometry as previously described (27). 
Briefly, following trypsinization and washing twice with phosphate-buffered saline (PBS) containing 3\% FBS, the cells were transferred into EP tubes (300 $\mu \mathrm{l} /$ tube). Each sample was then incubated with antibodies $(2 \mu \mathrm{l})$ against rat cluster of differentiation (CD)29 (cat.no. 555004; 1:100), CD34 (cat.no. 560518; 1:100), CD43 (cat. no. 553271; 1:100), CD90 (cat. no. 551401; 1:100) and CD105 (cat. no. 550546; 1:100) (all from BD Biosciences, San Jose, CA, USA) at $4^{\circ} \mathrm{C}$ for $1 \mathrm{~h}$ in the dark. A tube without antibodies served as a negative control. Finally, the samples were washed twice with PBS containing 3\% FBS and analyzed using a Beckman Coulter Epics XL flow cytometer (Beckman Coulter, Inc., Fullerton, CA, USA). Data were analyzed using Flowjo software v8.1 (Tree Star, Ashland, OR, USA).

Multiple differentiation analysis. The two types of BMSCs (P3) were plated into 6 -well dishes at a density of $5 \times 10^{5}$ cells/well and cultured in complete medium until the cells reached $80 \%$ confluence. To assess the ability of the BMSCs to form mineral nodules, the medium was changed to osteo-inductive medium [complete medium supplemented with $50 \mu \mathrm{g} / \mathrm{ml}$ vitamin $\mathrm{C}$, $10 \mathrm{nM}$ dexamethasone and $10 \mathrm{mM} \beta$-glycerophosphate (all Sigma-Aldrich; Merck KGaA)]; the medium was refreshed every 3 days. Following osteogenic induction for 14 or 21 days, the cells were fixed in $4 \%$ paraformaldehyde for $30 \mathrm{~min}$ at room temperature and stained with Alizarin Red S (Sigma-Aldrich; Merck KGaA) for $12 \mathrm{~h}$ at room temperature. Mineralization nodules were imaged using an inverted microscope. Furthermore, alkaline phosphatase (ALP) staining was conducted using a BCIP/NBT Alkaline Phosphatase Color Development kit (Beyotime Institute of Biotechnology), and ALP enzymatic activity was performed using an ALP detection kit (Nanjing Jiancheng Bioengineering Institute, Nanjing, China) according to the manufacturer's protocol. For adipogenic lineage differentiation, $80 \%$ confluent cells were induced using adipo-inductive medium [complete medium supplemented with $0.5 \mathrm{mM} 3$-isobutyl-1-methylxanthine, $1 \mu \mathrm{M}$ dexamethasone, $0.1 \mathrm{mM}$ indomethacin and $10 \mu \mathrm{g} / \mathrm{ml}$ insulin (all Sigma-Aldrich; Merck KGaA)]. The medium was refreshed every 3 days. After 7 and 14 days, the cells were fixed in $4 \%$ paraformaldehyde for $30 \mathrm{~min}$ at room temperature and stained with Oil Red O (SigmaAldrich; Merck KGaA). Photographic images were captured and the area of staining was measured using Image-Pro Plus 6.0 software (Media Cybernetics, Inc., Rockville, MD, USA).

Transwell migration assay. The chemotactic effects of SDF-1 $\alpha$ on cell migration were observed using a Transwell membrane system comprising a polycarbonate Transwell culture insert with an $8-\mu \mathrm{m}$ pore size (Corning Incorporated, Corning, NY, USA) in 24-well plates. Prior to testing in the migration assay, the cells were starved and incubated in $\alpha$-MEM without FBS. To identify a desirable concentration of SDF-1 $\alpha$ (Peprotech, Inc., Rocky Hill, NJ, USA) for cell migration, different SDF-1 $\alpha$ concentrations were used in the lower chambers of the system (total volume, $600 \mu \mathrm{l} /$ well). The medium used for this experiment was $\alpha$-MEM (supplemented with 5\% FBS) containing 0, 30, 50, 100 or $150 \mathrm{ng} / \mathrm{ml} \mathrm{SDF}-1 \alpha$. A total of $1 \times 10^{5}$ BMSCs (P3) were placed in each upper chamber following resuspension in $150 \mu \mathrm{l}$ $\alpha$-MEM containing 5\% FBS. The system was incubated for $6 \mathrm{~h}$ and fixed with $4 \%$ paraformaldehyde at $4^{\circ} \mathrm{C}$ for $20 \mathrm{~min}$. Each Transwell membrane was removed after stripping to detach non- migrated cells. Finally, the cells that had migrated to the lower surface of each membrane were stained with $0.2 \%$ crystal violet at $25^{\circ} \mathrm{C}$ (Sigma-Aldrich; Merck KGaA) for $1 \mathrm{~h}$, and cells in five random fields (x100 magnification) were counted for statistical analysis. Based on the data obtained from the cell migration assay using this Transwell membrane system, a concentration of $100 \mathrm{ng} /$ $\mathrm{ml} \mathrm{SDF}-1 \alpha$ was selected for all further experiments. To examine the effect of the presence of SDF-1 $\alpha$ on cell migration, prior to the migration assay, the BMSCs were incubated in $\alpha$-MEM without FBS but with a CXCR4 antagonist (AMD3100; Sigma-Aldrich; Merck $\mathrm{KGaA}$ ) at a concentration of $10 \mu \mathrm{g} / \mathrm{ml}$ for $2 \mathrm{~h}$.

Immunofluorescence staining. The CXCR4 expression profiles in the two types of BMSCs at two passages (P3 and P6) were assessed by immunofluorescence staining. Cells were collected and fixed in $4 \%$ paraformaldehyde at $4^{\circ} \mathrm{C}$ for $20 \mathrm{~min}$. Following treatment with $0.5 \%$ Triton $\mathrm{X}-100$ and $1 \%$ bovine serum albumin (Thermo Fisher Scientific, Inc.) for $1 \mathrm{~h}$ at $25^{\circ} \mathrm{C}$, cells were incubated with rabbit anti-CXCR4 antibody (ab124824; 1:100; Abcam, Cambridge, MA, USA) overnight at $4^{\circ} \mathrm{C}$. After washing with PBS, cells were incubated with Alexa Fluor ${ }^{\circledR}$ 488-conjugated anti-CXCR4 antibody (ab208128; Abcam) for $30 \mathrm{~min}$ at $37^{\circ} \mathrm{C}$ followed by DAPI (Sigma-Aldrich; Merck $\mathrm{KGaA}$ ) staining for $2 \mathrm{~min}$, to counterstain the cell nuclei. The sections were mounted and then observed under a Zeiss LSM 710 confocal microscope (Carl Zeiss AG, Oberkochen, Germany) and the intensity of the CXCR4-positive area were measured using Image-Pro Plus 6.0 software. The surface expression of CXCR4 was also analyzed using flow cytometric analysis as previously described (22). The cells were incubated with FITC-conjugated anti-CXCR4 antibody (Abcam).

Reverse transcription-quantitative polymerase chain reaction (RT-qPCR). After 21 or 14 days of osteogenic/adipogenic induction, the cells were harvested. The total RNA of the cells was extracted using TRIzol reagent and complementary DNA (cDNA) was obtained using SuperScript II reverse transcriptase (both Invitrogen; Thermo Fisher Scientific, Inc.) according to the manufacturer's protocol. Reverse transcription of the total RNA was performed using a RevertAid First Strand cDNA Synthesis kit (Takara Bio Inc., Otsu, Japan). qPCR was performed on 10-fold-diluted cDNA samples in double-distilled water using SYBR-Green Master (Roche Diagnostics GmbH, Mannheim, Germany) and a CFX Connect $^{\text {TM }}$ Real-Time PCR Detection system (Bio-Rad Laboratories, Inc.). Amplification was performed under the following conditions: Denaturation at $95^{\circ} \mathrm{C}$ for $3 \mathrm{~min}$, prior to 39 cycles at $95^{\circ} \mathrm{C}$ for $15 \mathrm{sec}$ and $60^{\circ} \mathrm{C}$ for $30 \mathrm{sec}$. The primers used for qPCR were: Runt-related transcription factor 2 (Runx2) forward, 5'-GCCACCTTCACTTACACCCC-3' and reverse, 5'-CGCTGACGAAGTACCATAGTAGAG-3'; osteocalcin (OCN) forward, 5'-AGACTCCGGCGCTACCTCAAC AAT-3' and reverse, 5'-CAGCTGTGCCGTCCATACT-3'; ALP forward, 5'-CTAGTTCCTGGGAGATGGTA-3' and reverse, 5'-GTGTTGTACGTCTTGGAGAGA-3'; peroxisome proliferator-activated receptor (PPAR) $\gamma$ forward, 5'-AGTGGA GACCGCCC-3' and reverse, 5'-GCAGCAGGTTGTCTT-3'; GAPDH forward, 5'-GGAAACCCATCACCATCTTC-3' and reverse, 5'-GCCATCCACAGTCTTCTGAGT-3'. The mRNA expression levels of the target genes were compared following 
normalization with the internal control GAPDH based on the $2^{-\Delta \Delta \mathrm{Cq}}$ method (28). In addition, the total RNA of the two groups of BMSCs at different passages (P3 and P6) was extracted for relative gene expression analysis by RT-qPCR based on the $2^{-\Delta \Delta \mathrm{Cq}}$ method. The target genes and primer sequences were as follows: CXCR4 forward, 5'-CCTCCTCCT GACTATCCCTGA-3' and reverse, 5'-CGAACTCACATC CTTGCTTG-3'; GAPDH forward, 5'-GGAAACCCATCA CCATCTTC-3' and reverse, 5'-GCCATCCACAGTCTTCTG AGT-3'. GAPDH served as an internal reference.

Protein isolation and western blot analysis. Western blot analysis was performed as previously described (22). Briefly, the two types of BMSCs at P3 and P6 were lysed using lysis buffer (Sigma-Aldrich; Merck KGaA) on ice following a standard procedure and then centrifuged at $16096 \mathrm{x}$ g for $10 \mathrm{~min}$ at $4^{\circ} \mathrm{C}$ to collect the total cell protein. The cell protein concentrations were determined using a BCA Protein Assay kit (Beyotime Institute of Biotechnology) according to the manufacturer's protocol. The proteins were subsequently denatured by boiling and resolved using $12 \%$ SDS-PAGE and transferred onto polyvinylidene difluoride membranes by electrophoretic separation. The membranes were blocked with $5 \%$ skimmed milk in TBS-T buffer $(25 \mathrm{~mm}$ Tris-HCl, $\mathrm{pH} 8.00,125 \mathrm{~mm} \mathrm{NaCl}$ and $0.1 \%$ Tween-20) for $1 \mathrm{~h}$ at room temperature. The membranes were then incubated overnight with primary antibodies at $4^{\circ} \mathrm{C}$. The primary antibodies included anti-CXCR4 (ab124824; 1:500; Abcam) and anti-GAPDH (ab8245; 1:1000; Abcam), anti-AKT (cat. no. 9272; 1:500; Cell Signaling Technology, Danvers, MA, USA) and anti-p-AKT (cat. no. 9271; 1:500; Cell Signaling Technology). The membranes were washed three times with TBS-T to remove the excess antibodies prior to incubation with anti-rabbit secondary antibodies (A0192; 1:8,000; Beyotime Institute of Biotechnology) conjugated with horseradish peroxidase. The proteins were detected by enhanced chemiluminescence (SigmaAldrich; Merck KGaA). GAPDH was used as an internal control to normalize the samples. To investigate the signal transduction mechanisms involved in SDF-1 $\alpha$-induced chemotactic activity, total AKT (t-AKT) and phosphorylated AKT (p-AKT) protein levels were analyzed using western blot analysis in the two sets of BMSCs. Prior to protein isolation and western blot analysis, the two sets of BMSCs were treated with $100 \mathrm{ng} / \mathrm{ml} \mathrm{SDF}-1 \alpha$ for $5,15,30,60$ or $120 \mathrm{~min}$. To further examine the effects of the presence of SDF-1 $\alpha / \mathrm{CXCR} 4$ on $\mathrm{p}-\mathrm{AKT}$, prior to the treatment with SDF-1 $\alpha$, the BMSCs were incubated in $\alpha$-MEM without FBS but with CXCR4 antagonist at a concentration of $10 \mu \mathrm{g} / \mathrm{ml}$ for $2 \mathrm{~h}$. The two sets of BMSCs were then treated with $100 \mathrm{ng} / \mathrm{ml}$ SDF-1 $\alpha$ for $5,15,30$ or 60 min.

Statistical analysis. Results are presented as the mean \pm SD and all experiments were repeated three times. Data were analyzed by one-way analysis of variance (ANOVA) followed by Tukey's post hoc test using SPSS 18.0 software (SPSS, Inc., Chicago, IL, USA). $\mathrm{P}<0.05$ was considered to indicate a statistically significant difference for all tests.

\section{Results}

Cell culture and general characterization. As shown in Fig. 1, the BMC, BMD, BV/TV and Max load in the OVX group were significantly lower compared with those in the Sham group $(\mathrm{P}<0.05)$, indicating that the rats in OVX-group had poorer bone tissue-forming ability compared with those in the Sham group. In addition, the rats in the OVX group had a BMD of $>2.5$ SDs below the mean BMD of the Sham group, suggesting the PMO rat model was successfully established (25). It was observed that BMSCs at P0 adhered to the culture plates and had a mixture of round and spindle-like morphology, whereas cells at P2 exhibited normal growth and a typical spindle-like appearance (Fig. 2A). However, no marked difference was observed between the BMSCs from the Sham and OVX groups at each time point. In addition, no significant difference in colony-forming ability was detected between the two sets of cells (Fig. 2B and C). However, OVX-BMSCs exhibited a stronger proliferative ability than Sham-BMSCs on days 4, 6, 9 and 10- (P<0.05; Fig. 2D). The results of the immunophenotypic analysis revealed that the two sets of BMSCs exhibited similar patterns of surface molecule expression; specifically, they were positive for MSC-specific surface markers (CD29, CD90 and CD105) and negative for hematopoietic cell markers (CD34 and CD45; Fig. 2E).

Differentiation potential analysis. The multiple differentiation potentials of the two groups of BMSCs were determined using osteogenic and adipogenic differentiation assays (Fig. 3). The osteogenic ability was indicated by the area of Alizarin Red S staining (for mineralized nodules) following osteogenic induction (Fig. 3A-C). Quantitative analysis revealed that the Sham-BMSCs produced a larger area of mineralized nodules than the OVX-BMSCs at 14 and 21 days $(\mathrm{P}<0.05)$. Subsequently, ALP was detected using an ALP color development kit. Consistent with the observed Alizarin Red S staining results on days 7 and 14, the ALP staining results indicated that OVX-BMSCs displayed less osteogenic differentiation than Sham-BMSCs (Fig. 3D and E). In order to observe the dynamic changes of ALP in the two cell types, ALP activity was detected. In accordance with the above osteogenic induction results, OVX-BMSCs exhibited reduced ALP activity, indicative of impaired osteogenic ability, at all time points, although there was only a statistically significant difference on days 6,8 , 12 and $14(\mathrm{P}<0.05 ;$ Fig. 3F). Such a trend was further confirmed by the results of RT-qPCR. Following osteogenic induction, it was detected that the expression levels of osteogenic marker genes (Runx2, OCN and ALP) in the Sham-BMSCs group were significantly higher than those in the OVX-BMSCs group $(\mathrm{P}<0.05$; Fig. 3J). Following adipogenic induction, Oil Red O-positive lipid droplets were observed in the two cell lines, indicating that the two types of BMSCs possessed adipogenic differentiation potential. However, the OVX-BMSCs exhibited stronger adipogenic activity with a significantly larger area of lipid droplets following Oil Red $\mathrm{O}$ staining on day 14 (P<0.05; Fig. 3G-I). Likewise, OVX-BMSCs had a higher mRNA level of the adipogenic differentiation-associated gene PPAR $\gamma$ compared with the Sham-BMSCs ( $\mathrm{P}<0.05$; Fig. 3K).

Chemotactic activity assay. The results of the in vitro cell chemotaxis and chemotaxis-blocking assays demonstrated that the migration of BMSCs was dependent upon the concentration of SDF-1 $\alpha$, with a peak migration rate at $100 \mathrm{ng} / \mathrm{ml}$ in the two sets of BMSCs. Therefore, a concentration of $100 \mathrm{ng} / \mathrm{ml} \mathrm{SDF}-1 \alpha$ 

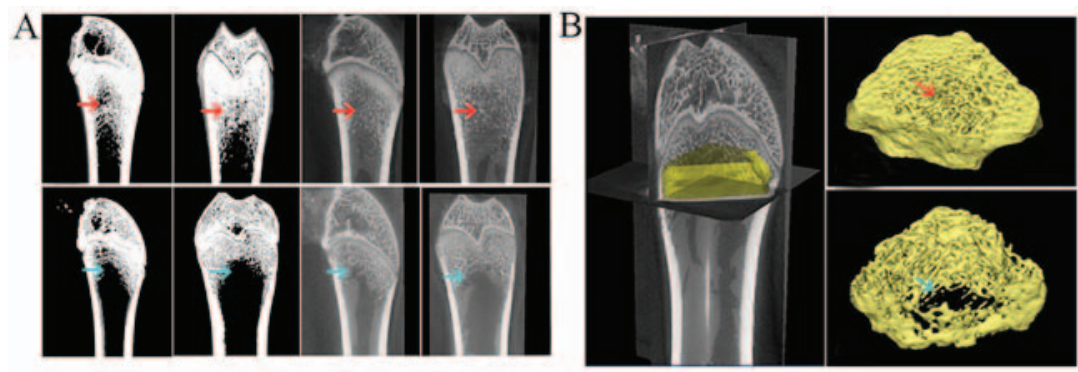

$\mathrm{C}$

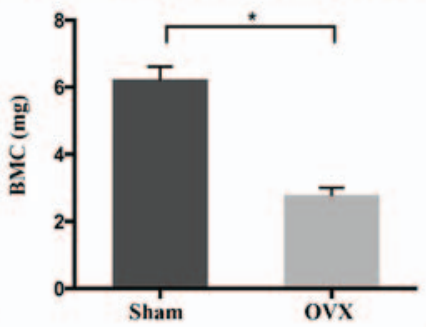

$\mathrm{E}$

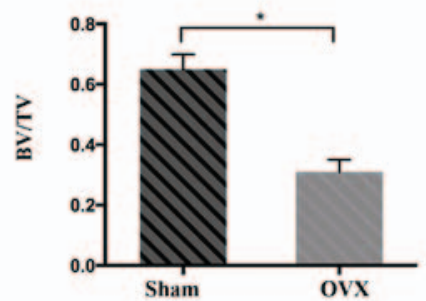

D

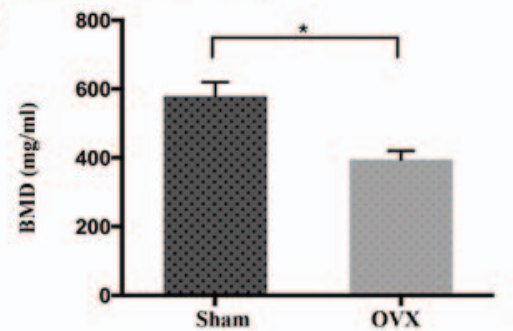

$\mathrm{F}$

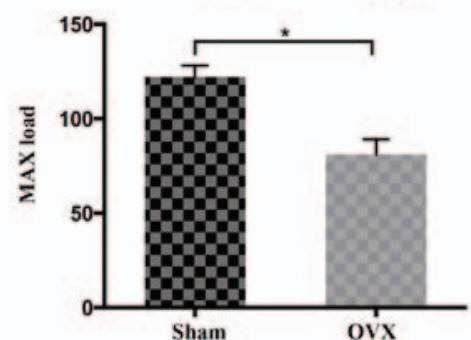

Figure 1. Identification of the establishment of a rat model of postmenopausal osteoporosis via micro-CT analysis. (A) Representative coronary and sagittal reconstruction images obtained by the micro-CT analysis of rat femoral bone from the Sham and OVX groups. (B) Three-dimensional reconstruction area of rat femoral bone to analyze the bone density index as follows: Red arrows indicate that rats in the Sham group had dense femoral cortical bone, as well as continuous and compact cancellous bone; while the blue arrows indicate that multiple cavities were present in the cancellous bone in the OVX group with trabecular bone fracture and lower bone density. Quantitative comparison of the (C) BMC, (D) BMD, (E) BV/TV and (F) Max load between the Sham and OVX groups. Data are presented as the mean \pm standard deviation. " $\mathrm{P}<0.05$ as indicated $(\mathrm{n}=8)$. Micro-CT, micro-computed tomography; OVX, ovariectomy; $\mathrm{BMC}$, bone mineral content; $\mathrm{BMD}$, bone mineral density; $\mathrm{BV} / \mathrm{TV}$, ratio of bone volume to total volume.
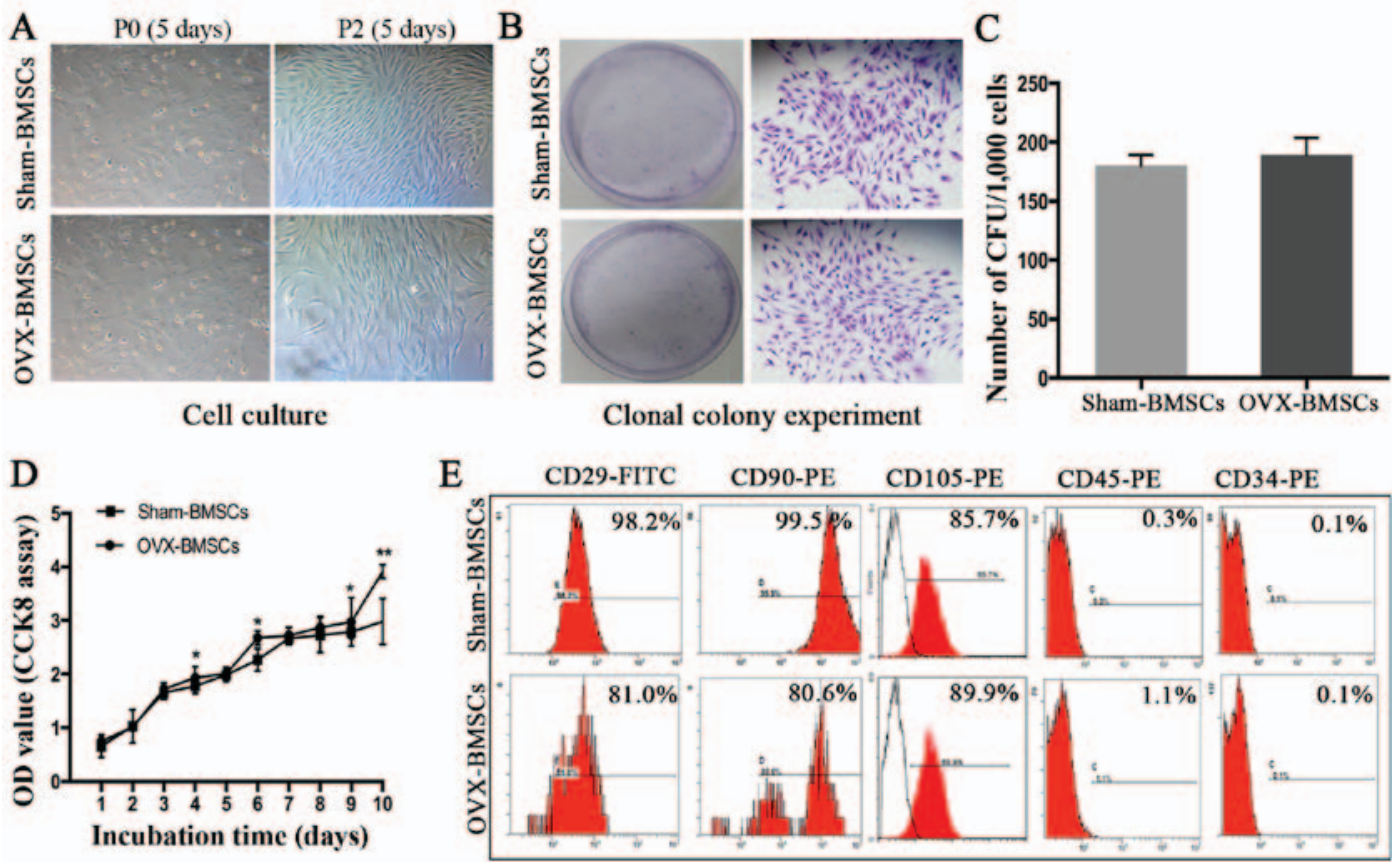

Figure 2. Isolation and characterization of Sham-BMSCs and OVX-BMSCs. (A) Representative images of phase-contrast photomicrographs of primary cells at P0 and P2 (x40 magnification). (B) Representative images of the number of CFUs formed by Sham-BMSCs and OVX-BMSCs following culture for 12 days (x40 magnification). (C) Quantitative analysis of the number of CFUs formed by Sham-BMSCs and OVX-BMSCs (n=7). (D) Proliferative capacity of Sham-BMSCs and OVX-BMSCs evaluated by CCK-8 assay (n=7). (E) Representative images of cell surface marker expression in Sham-BMSCs and OVX-BMSCs. Data are shown as the mean \pm standard deviation. " $\mathrm{P}<0.05$ for Sham-BMSCs vs. OVX-BMSCs. BMSCs, bone marrow mesenchymal stem cells; OVX, ovariectomy; P0, passage 0; P2, passage 2; CFU, colony-forming unit; CCK-8, Cell Counting kit-8; OD, optical density; CD, cluster of differentiation. 

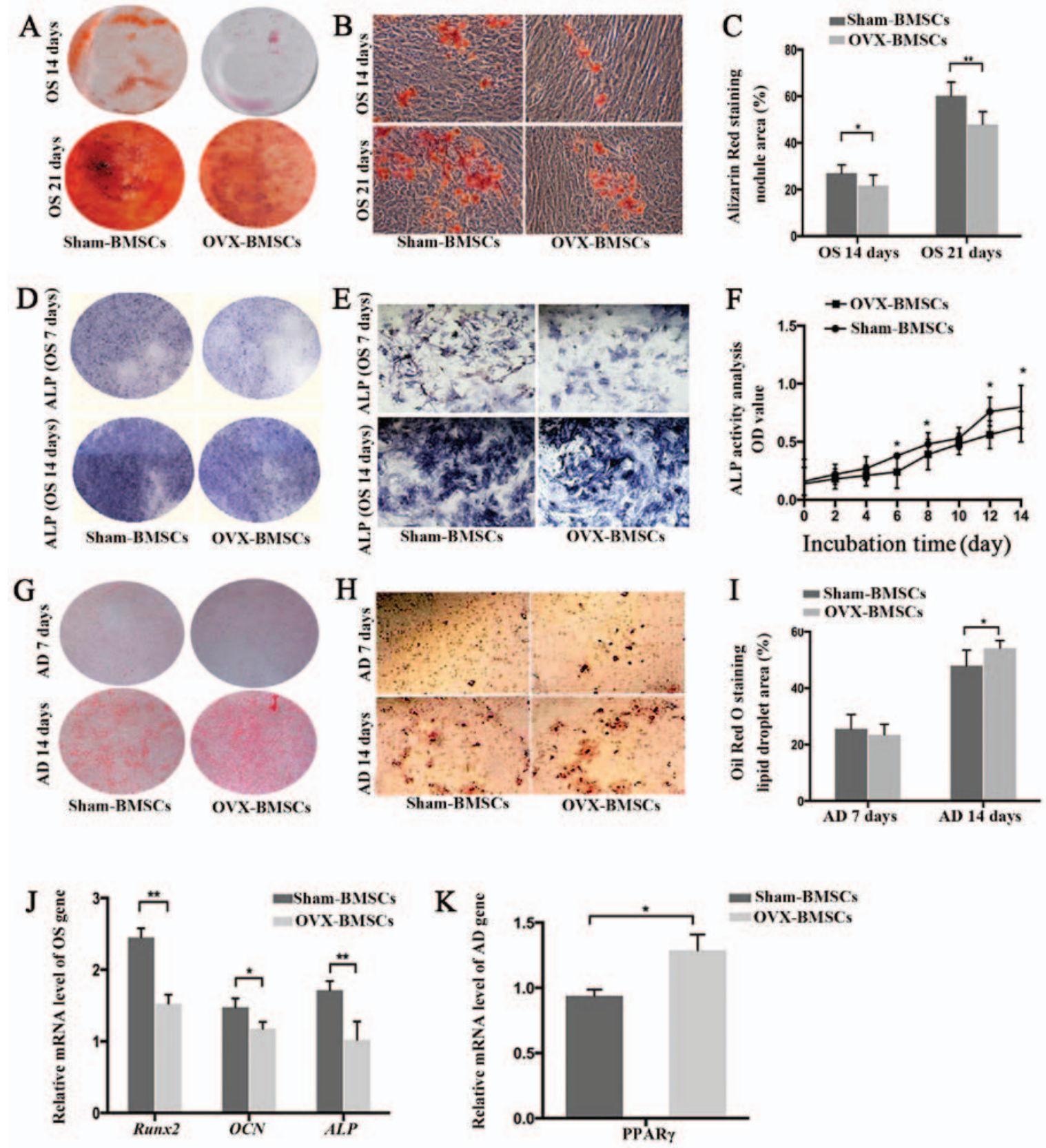

Figure 3. Differentiation potential of Sham-BMSCs and OVX-BMSCs. (A and B) Representative images of Alizarin Red S-stained mineral deposits following 14 or 21 days of osteogenic induction (A, general view; B, x40 magnification) and (C) quantification of the area of dye absorption for Alizarin Red S in the Sham-BMSCs and OVX-BMSCs. (D and E) Representative images of ALP staining following 7 or 14 days of osteogenic induction (D, general view; E, x200 magnification) and (F) quantification of ALP activity as different time points in the Sham-BMSCs and OVX-BMSCs. (G and H) Representative images of Oil Red O-stained lipid inclusions following 7 and 14 days of adipogenic induction (G, general view; $\mathrm{H}$, x200 magnification) and (I) quantification of the area of dye absorption for Oil Red O in Sham-BMSCs and OVX-BMSCs. (J) Reverse transcription-quantitative polymerase chain reaction measurements of OCN, Runx2 and ALP genes following osteogenic induction. The values are ratios relative to GAPDH ( $\mathrm{n}=4)$. (K) Relative levels of expression of the PPAR $\gamma$ gene following 14 days of osteogenic induction $(n=4)$. Data are presented as the mean \pm standard deviation. ${ }^{*} \mathrm{P}<0.05$ and ${ }^{* *} \mathrm{P}<0.01$ for Sham-BMSCs vs. OVX-BMSCs. BMSCs, bone marrow mesenchymal stem cells; OVX, ovariectomy; ALP, alkaline phosphatase; OS, osteogenic; AD, adipogenic; RunX2, Runt-related transcription factor 2; OCN, osteocalcin; ALP, alkaline phosphatase;PPAR, peroxisome proliferator-activated receptor.

was selected for all further experiments. It was observed that the presence of the CXCR4 antagonist AMD3100 greatly decreased the migration activity of Sham-BMSCs and OVX-BMSCs to nearly half of that for the respective BMSCs treated with SDF-1 $\alpha$ without antagonist. In addition, the OVX-BMSCs exhibited significantly impaired chemotactic activity compared with the Sham-BMSCs ( $\mathrm{P}<0.05$; Fig. 4A), with the exception of when $100 \mathrm{ng} / \mathrm{ml}$ SDF-1 $\alpha$ plus AMD3100 was used.
Temporal changes in CXCR4 expression. The expression of CXCR4 on the cell surface was determined using flow cytometric analysis. The results revealed that the Sham-BMSCs exhibited significantly higher CXCR4 expression compared with the OVX-BMSCs ( $\mathrm{P}<0.05$; Fig. 4B and $\mathrm{C})$. To compare the expression of CXCR4 in the two sets of BMSCs, the expression of CXCR4 was detected using an immunofluorescence, the CXCR4 mRNA level was analyzed by RT-qPCR and the CXCR4 protein level was analyzed using western blot analysis. At the same cell 

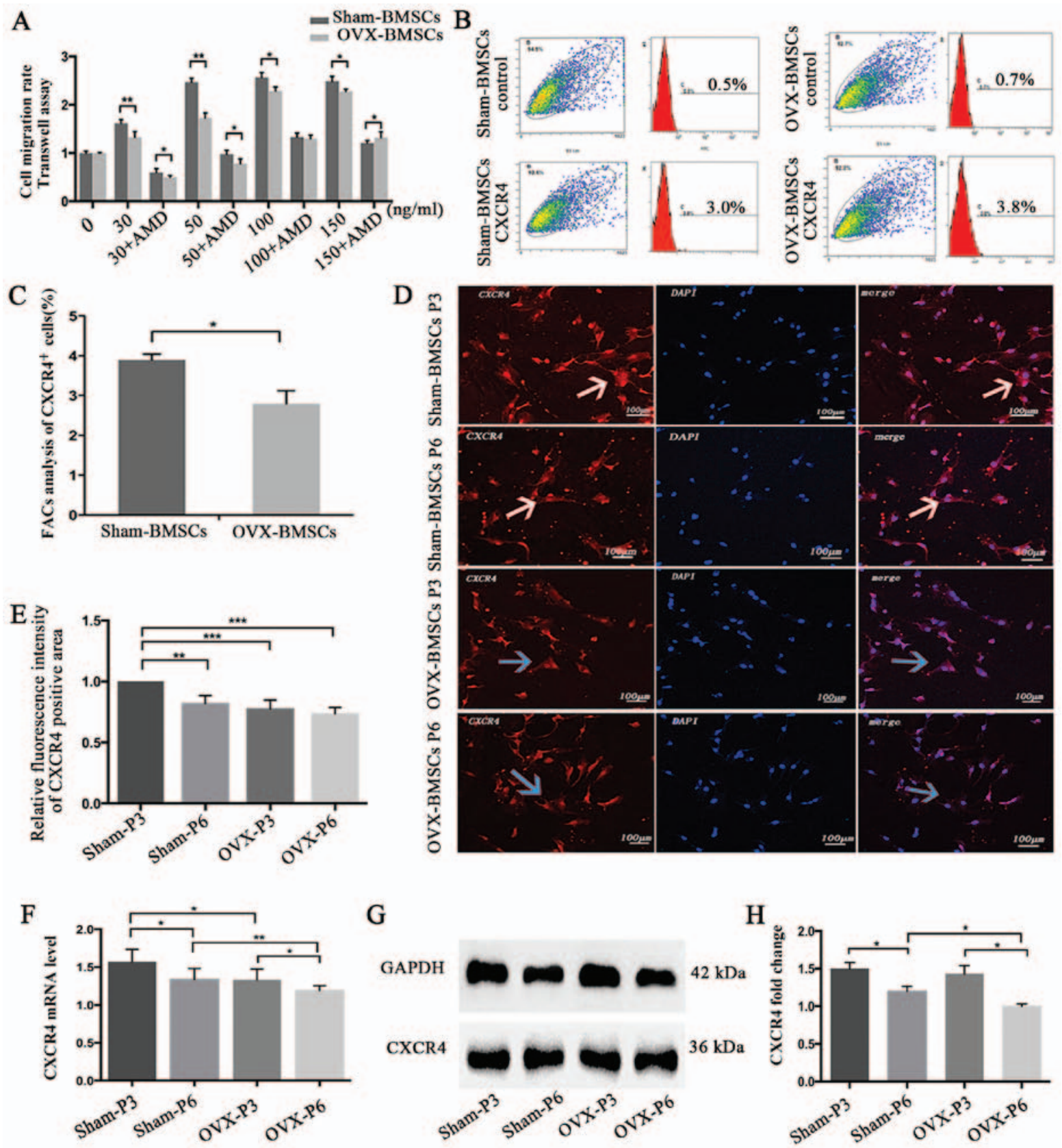

Figure 4. Chemotaxis assay and CXCR4 expression analysis of Sham-BMSCs and OVX-BMSCs. (A) Quantification of the chemotactic activity of Sham-BMSCs and OVX-BMSCs towards SDF-1 $\alpha$ in the absence or presence of AMD3100. Culture medium with different concentrations of SDF-1 $\alpha$ (30,50, 100 or 150 ng/ml) was applied to the lower chamber of the Transwell system. (B) Representative images showing the detection of surface CXCR4 expression by flow cytometry in Sham-BMSCs and OVX-BMSCs $(\mathrm{n}=5)$ and $(\mathrm{C})$ data analysis of the percentage of CXCR4-positive cells. (D) Representative immunofluorescent images of CXCR4 staining on Sham-BMSC and OVX-BMSC (P3 and P6) membranes (x200 magnification) and (E) quantitative analysis of CXCR4 fluorescence intensity. White arrows indicate higher expression of CXCR4, whereas blue arrows indicate lower expression of CXCR4 with weaker red fluorescence. (F) CXCR4 expression was analyzed by reverse transcription-quantitative polymerase chain reaction and (G and $\mathrm{H}$ ) western blot analysis in Sham-BMSCs and OVX-BMSCs (P3 and P6). (G) Representative blots and (H) quantified data, presented as the mean \pm standard deviation. "P $<0.05$ between the groups as indicated. CXCR4, CXC chemokine receptor type 4; BMSCs, bone marrow mesenchymal stem cells; OVX, ovariectomy; SDF-1 $\alpha$, stromal cell-derived factor- $1 \alpha ; \mathrm{P} 3$, passage 3; P6, passage 6

passage, the CXCR4 immunofluorescence, mRNA and protein level in the Sham-BMSCs were much higher than those in the OVX-BMSCs, though no statistical significance for CXCR4 immunofluorescence was noticed at P6 (Fig. 4D-H). In addition, the results indicated that cell passage was likely to adversely affect the expression of CXCR4, since the Sham-BMSCs and OVX-BMSCs exhibited higher CXCR4 immunofluorescence, mRNA and protein levels at P3 than at P6 (Fig. 4D-H).
$p$-AKT analysis. To further investigate the signal transduction mechanisms involved in the SDF- $1 \alpha$-induced chemotactic activity of BMSCs from the PMO rat model, t-AKT and p-AKT protein levels were analyzed at different time points as the duration of stimulation increased using western blot analysis in the two sets of BMSCs (Fig. 5). SDF-1 $\alpha$ significantly increased the protein levels of p-AKT at $30 \mathrm{~min}$, which then started to decline at $120 \mathrm{~min}$ in the Sham-BMSCs. However, 

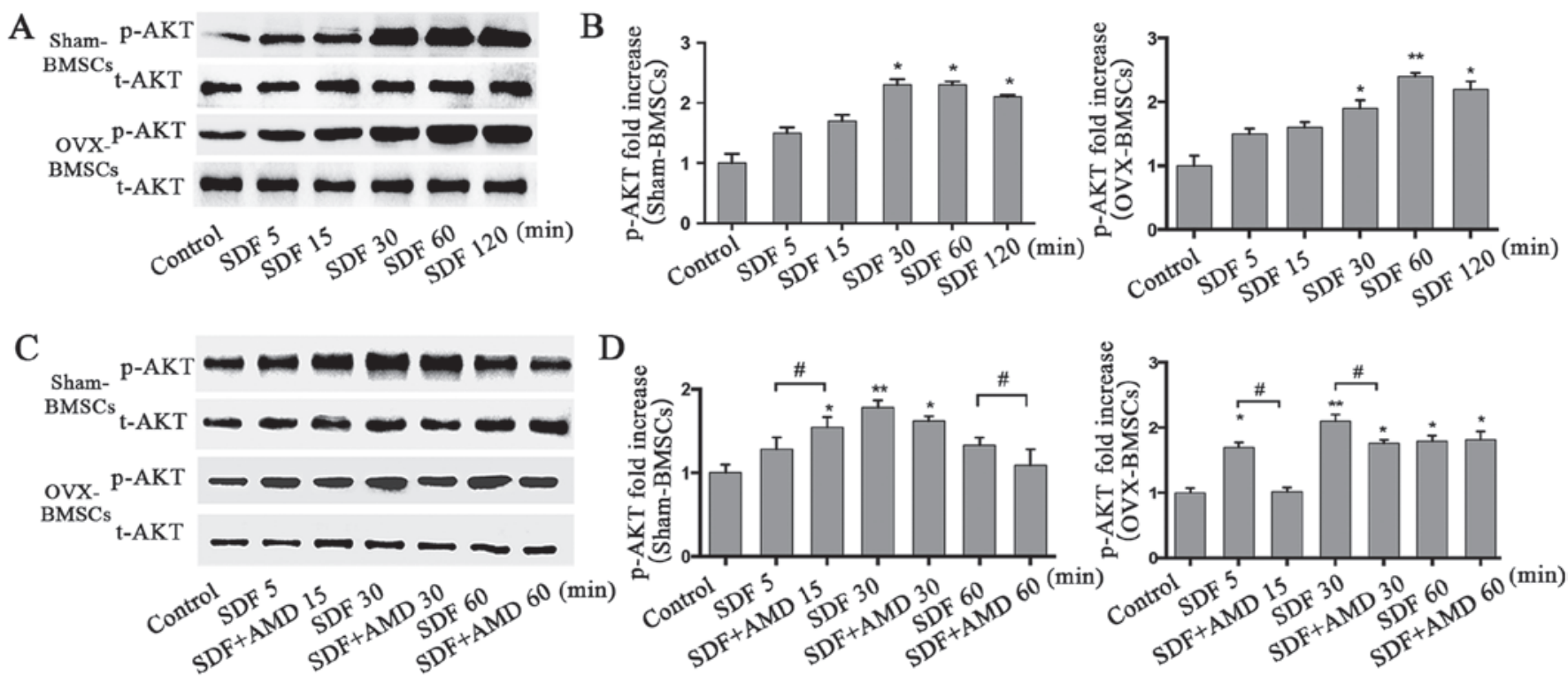

Figure 5. Analysis of p-AKT in Sham-BMSCs and OVX-BMSCs by western blot analysis. (A) Representative images of the western blot analysis of t-AKT and p-AKT in Sham-BMSCs and OVX-BMSCs following stimulation with SDF-1 $\alpha$ (100 ng/ml) for different time periods $(5,15,30,60$ or $120 \mathrm{~min})$. (B) Quantitative analysis of the relative protein level of p-AKT. (C) Representative images of the western blot analysis of t-AKT and p-AKT in Sham-BMSCs and OVX-BMSCs following stimulation with SDF-1 $\alpha(100 \mathrm{ng} / \mathrm{ml})$ in the presence of AMD3100 for different durations $(5,15,30$ or $60 \mathrm{~min})$. (D) Quantitative analysis of the relative protein level of $\mathrm{p}-\mathrm{AKT}$. (A and C) The values are ratios relative to t-AKT. Data are presented as the mean \pm standard deviation. " $\mathrm{P}<0.05$ and ${ }^{* *} \mathrm{P}<0.01 \mathrm{vs}$. the control group; ${ }^{\#} \mathrm{P}<0.05$ as indicated. p-AKT, phosphorylated AKT; t-AKT, total AKT; BMSCs, bone marrow mesenchymal stem cells; OVX, ovariectomy; stromal cell-derived factor- $1 \alpha$.

for OVX-BMSCs, the level of p-AKT peaked at $60 \mathrm{~min}$ and then declined at $120 \mathrm{~min}$ (Fig. 5A and B). As shown in Fig. 5C and D, BMSCs pretreated with AMD3100 had reduced levels of p-AKT compared with those that were not pretreated, but exhibited a similar trend.

\section{Discussion}

Osteoporosis is a common condition in postmenopausal women, which predisposes individuals to an increased risk of bone fragility and bone fractures. It is considered that in individuals with osteoporosis, bone loss may be attributed to changes in the osteogenic differentiation and adipocyte ability of BMSCs $(11,29)$. In the present study, the general biological characteristics of Sham-BMSCs and OVX-BMSCs were compared. No marked difference between the two groups of BMSCs was detected in terms of cell culture and the expression of stem cell surface markers; however, OVX-BMSCs exhibited a greater ability to proliferate. Furthermore, osteoporosis was indicated to impair osteogenic ability since a smaller area of mineralized nodules and lower ALP activity, as well as lower expression levels of osteogenic marker genes (Runx2, OCN and ALP) were observed for OVX-BMSCs compared with Sham-BMSCs. These results are in accordance with those of Wu et al (29) and Yang et al (30) who reported that ALP activity was more active in Sham-BMSCs than in OVX-BMSCs $(29,30)$. However, the OVX-BMSCs exhibited stronger adipogenic activity than Sham-BMSCs in the present study, since a larger lipid droplet area with Oil Red O staining as well as a higher expression level of the adipogenic differentiation-associated gene PPAR $\gamma$ was observed. These results are consistent with those in the studies described by Pino et al (11). In the pathogenesis of osteoporosis, the impairment of the functionality and microenvironment of MSCs results in the dysregulation of bone turnover, such that bone resorption exceeds bone formation (11). The OVX-BMSCs exhibited intrinsic functional alterations, leading to poor osteogenic capability and increased adipogenesis. The osteoporotic bone marrow microenvironment differs from the normal microenvironment in that it has increased concentrations of pro-adipogenic and pro-inflammatory regulatory factors (31). The content and/or quality of adipocytes in the bone marrow appears critical to the impairment of BMSCs; thus, osteoporosis may be comparable with other age-associated diseases, such as obesity, atherogenesis and diabetes, which are characterized by unbalanced extramedullary adipocyte formation and signaling $(11,32,33)$.

The SDF-1 $\alpha /$ CXCR4 axis is considered to be the key pathway influencing the homing capacity of BMSCs. It has been demonstrated that the augmentation of SDF-1 $\alpha$ at the injury site or transplantation of MSCs that overexpress CXCR4 may improve outcomes $(7,34)$. However, to the best of our knowledge, whether osteoporosis affects the chemotaxis of BMSCs towards SDF-1 $\alpha$ has not been elucidated. In the present study, it was observed that SDF-1 $\alpha$ concentration-dependently increased the migration of OVX-BMSCs and Sham-BMSCs within a limited concentration range, whereas such migration was inhibited by AMD3100, an antagonist of CXCR4. Notably, Sham-BMSCs exhibited greater migration than OVX-BMSCs at nearly all tested concentrations of SDF-1 $\alpha$, regardless of AMD3100, suggesting that osteoporosis negatively affected SDF-1 $\alpha$-directed BMSC migration. The surface expression level of CXCR4 on BMSCs has been indicated to determine their efficiency of homing and the regeneration response to target tissues $(35,36)$. Wynn et al $(37)$ found that the proportion human MSCs with expression of the CXCR4 receptor on the 
cell membrane ranged from 1 to $3.9 \%$. Similarly, the flow cytometric analysis in the present study demonstrated that the proportion of BMSCs positive for the expression of CXCR4 in the Sham- and OVX-BMSCs was no more than $4 \%$. However, the percentage of CXCR4-positive cells was higher in the Sham-BMSCs group compared with the OVX-BMSCs group. To further investigate the possible mechanism underlying the reduced migration of OVX-BMSCs towards SDF-1 $\alpha$, CXCR4 expression, mRNA levels and protein levels were analyzed. The CXCR4 immunofluorescence intensity, and mRNA and protein expression levels in the Sham-BMSCs were higher than those in the OVX-BMSCs. The lower expression of CXCR4 in the OVX-BMSCs may partly explain their lower chemotactic migration ability. By contrast, previous studies have found that in certain acute disease models, such as rat abdominal aortic aneurysm model (38) and severe acute pancreatitis model (39), the mRNA and protein levels of CXCR4 in BMSCs were much higher than those in the BMSCs from the normal animals. These phenomena may result from an increase in the number of BMSCs expressing CXCR4 as well as promotion of the autocrine and paracrine activity of SDF-1 $\alpha$ in wounds or tissues affected by acute inflammation. Signaling by SDF-1 $\alpha$ stimulates the expression of CXCR4 on the cell surface and promotes the homing of BMSCs towards defects; however, as soon as the cells reach their destination, the expression of CXCR4 is reduced to a low level (40). In order to further investigate the expression of CXCR4 in BMSCs, cell passage was investigated as an influential factor in the present study, since BMSCs have highly multi-directional differentiation potential during culture and cell passage. In the present study, a decline in CXCR4 mRNA and protein expression was detected when the Sham-BMSCs and OVX-BMSCs were cultured from P3 to P6. It has been demonstrated that the expression of CXCR4 in normal human BMSCs can be greatly attenuated during cell passage $(41,42)$. However, to the best of our knowledge, the present study is the first to demonstrate this in rat Sham-BMSCs and OVX-BMSCs. Therefore, this observation combined with the recommendations of previous researchers who identified that cells at an early passage exhibit better stability, growth rates and more potent anti-inflammatory activity, indicates that BMSCs of passage 2-4 are likely to be the most effective for the repair of tissue defects $(41,43,44)$.

The AKT signaling pathway is known to mediate the migration of cells induced by cytokines such as SDF-1 $\alpha$ in various types of cells (45-47). Therefore, the protein levels of $\mathrm{t}$-AKT and p-AKT were analyzed by western blot analysis in the present study. SDF-1 $\alpha(100 \mathrm{ng} / \mathrm{ml})$ induced time-dependent increases of p-AKT protein levels in Sham-BMSCs and OVX-BMSCs $(46,48)$. These results concur with those of other studies, which indicated that the AKT signaling pathway is involved in the induction of BMSC migration by SDF-1 $1 \alpha$. The activation of AKT has been suggested to serve multiple functions in the process of BMSC migration. For example, it may increase the secretion of actin and myosin, which are considered to be associated with BMSC movement (49). In addition, it may contribute to the secretion of matrix metalloproteinase to promote BMSC migration via degradation of the extracellular matrix and basilar membrane (50). The results of the present study revealed that the time point at which the p-AKT levels peaked in the two types of BMSCs was significantly different, and was later in the OVX-BMSCs. These results are in accordance with those of Shahzad et al (51). The weaker sensitivity of p-AKT in OVX-BMSCs may be attributed to their lower expression of CXCR4 (52). The cells pretreated with AMD3100 exhibited lower protein levels of p-AKT than the cells that were not pretreated. These results are in accordance with dose-dependent increase in the migration of the BMSCs induced by SDF-1 $\alpha$ and the inhibition of the migration by AMD3100, suggesting that the reduced chemotaxis towards SDF- $1 \alpha$ of OVX-BMSCs may be associated with reduced sensitivity of p-AKT.

In conclusion, the general and SDF-1 $\alpha /$ CXCR4-related biological characteristics of OVX-BMSCs and Sham-BMSCs were investigated and compared, and their molecular mechanisms were evaluated. Compared with Sham-BMSCs, the OVX-BMSCs exhibited increased proliferation ability and impaired osteogenic ability as well as lower chemotactic activity towards SDF- $1 \alpha$, which may be partially associated with lower expression of CXCR4 and restricted AKT phosphorylation.

\section{References}

1. Baccaro LF, Conde DM, Costa-Paiva L and Pinto-Neto AM: The epidemiology and management of postmenopausal osteoporosis: A viewpoint from Brazil. Clin Interv Aging 10: 583-591, 2015.

2. Diab DL and Watts NB: Postmenopausal osteoporosis. Curr Opin Endocrinol Diabetes Obes 20: 501-509, 2013.

3. Shang F, Ming L, Zhou Z, Yu Y, Sun J, Ding Y and Jin Y: The effect of licochalcone A on cell-aggregates ECM secretion and osteogenic differentiation during bone formation in metaphyseal defects in ovariectomized rats. Biomaterials 35: 2789-2797, 2014.

4. No authors listed: NIH Consensus Development Panel on Osteoporosis Prevention, Diagnosis, and Therapy, March 7-29, 2000: highlights of the conference. South Med J 94: 569-573, 2001.

5. Riggs BL, Khosla S and Melton LJ III: Sex steroids and the construction and conservation of the adult skeleton. Endocr Rev 23: 279-302, 2002.

6. Pacifici R: Cytokines, estrogen, and postmenopausal osteoporosis - the second decade. Endocrinology 139: 2659-2661, 1998.

7. Anbinder AL, Moraes RM, Lima GM, Oliveira FE, Campos DR, Rossoni RD, Oliveira LD, Junqueira JC, Ma Y and Elefteriou F: Periodontal disease exacerbates systemic ovariectomy-induced bone loss in mice. Bone 83: 241-247, 2016.

8. Shoback D: Update in osteoporosis and metabolic bone disorders. J Clin Endocrinol Metab 92: 747-753, 2007.

9. Xiao Y, Wang Y, Li L, Li YH, Pang Y, Song JY and Jiang ZJ: Homing of chloromethylbenzoyl ammonia-labeled bone marrow mesenchymal stem cells in an immune-mediated bone marrow failure mouse model in vivo. Genet Mol Res 13: 11-21, 2014.

10. Yagi H, Soto-Gutierrez A, Parekkadan B, Kitagawa Y, Tompkins RG, Kobayashi N and Yarmush ML: Mesenchymal stem cells: mechanisms of immunomodulation and homing. Cell Transplant 19: 667-679, 2010.

11. Pino AM, Rosen CJ and Rodríguez JP: In osteoporosis, differentiation of mesenchymal stem cells (MSCs) improves bone marrow adipogenesis. Biol Res 45: 279-287, 2012.

12. Lau TT and Wang DA: Stromal cell-derived factor-1 (SDF-1): homing factor for engineered regenerative medicine. Expert Opin Biol Ther 11: 189-197, 2011.

13. Orimo A, GuptaPB,Sgroi DC,Arenzana-Seisdedos F, Delaunay T, Naeem R, Carey VJ, Richardson AL and Weinberg RA: Stromal fibroblasts present in invasive human breast carcinomas promote tumor growth and angiogenesis through elevated SDF-1/CXCL12 secretion. Cell 121: 335-348, 2005.

14. Bleul CC, Fuhlbrigge RC, Casasnovas JM, Aiuti A and Springer TA: A highly efficacious lymphocyte chemoattractant, stromal cellderived factor 1 (SDF-1). J Exp Med 184: 1101-1109, 1996.

15. Gupta SK, Lysko PG, Pillarisetti K, Ohlstein E and Stadel JM: Chemokine receptors in human endothelial cells. Functional expression of CXCR4 and its transcriptional regulation by inflammatory cytokines. J Biol Chem 273: 4282-4287, 1998. 
16. Ji JF, He BP, Dheen ST and Tay SS: Interactions of chemokines and chemokine receptors mediate the migration of mesenchymal stem cells to the impaired site in the brain after hypoglossal nerve injury. Stem Cells 22: 415-427, 2004.

17. Herberg S, Fulzele S, Yang N, Shi X, Hess M, PeriyasamyThandavan S, Hamrick MW, Isales CM and Hill WD: Stromal cell-derived factor-1 $\beta$ potentiates bone morphogenetic protein2 -stimulated osteoinduction of genetically engineered bone marrow-derived mesenchymal stem cells in vitro. Tissue Eng Part A 19: 1-13, 2013.

18. Herberg S, Kondrikova G, Periyasamy-Thandavan S, Howie RN, Elsalanty ME, Weiss L, Campbell P, Hill WD and Cray JJ: Inkjet-based biopatterning of SDF-1 $\beta$ augments BMP2-induced repair of critical size calvarial bone defects in mice. Bone 67 : 95-103, 2014

19. Yang D, Sun S, Wang Z, Zhu P, Yang Z and Zhang B: Stromal cell-derived factor-1 receptor CXCR4-overexpressing bone marrow mesenchymal stem cells accelerate wound healing by migrating into skin injury areas. Cell Reprogram 15: 206-215, 2013.

20. Kitaori T, Ito H, Schwarz EM, Tsutsumi R, Yoshitomi H, Oishi S, Nakano M, Fujii N, Nagasawa T and Nakamura T: Stromal cell-derived factor 1/CXCR 4 signaling is critical for the recruitment of mesenchymal stem cells to the fracture site during skeletal repair in a mouse model. Arthritis Rheum 60: 813-823, 2009.

21. Lapidot T, Dar A and Kollet O: How do stem cells find their way home? Blood 106: 1901-1910, 2005.

22. Shao H, Xu Q, Wu Q, Ma Q, Salgueiro L, Wang J, Eton D, Webster KA and Yu H: Defective CXCR4 expression in aged bone marrow cells impairs vascular regeneration. J Cell Mol Med 15: 2046-2056, 2011.

23. Guang LG, Boskey AL and Zhu W: Age-related CXC chemokine receptor-4-deficiency impairs osteogenic differentiation potency of mouse bone marrow mesenchymal stromal stem cells. Int J Biochem Cell Biol 45: 1813-1820, 2013.

24. Wronski TJ, Lowry PL, Walsh CC and Ignaszewski LA: Skeletal alterations in ovariectomized rats. Calcif Tissue Int 37 324-328, 1985.

25. Namkung-Matthai H, Appleyard R, Jansen J, Hao Lin J, Maastricht S, Swain M, Mason RS, Murrell GA, Diwan AD and Diamond T: Osteoporosis influences the early period of fracture healing in a rat osteoporotic model. Bone 28: 80-86, 2001.

26. Lennon DP and Caplan AI: Isolation of rat marrow-derived mesenchymal stem cells. Exp Hematol 34: 1606-1607, 2006.

27. Wen L, Wang Y, Wen N, Yuan G, Wen M, Zhang L, Liu Q, Liang Y, Cai C, Chen X, et al: Role of endothelial progenitor cells in maintaining stemness and enhancing differentiation of mesenchymal stem cells by indirect cell-cell interaction. Stem Cells Dev 25: 123-138, 2016.

28. Livak KJ and Schmittgen TD: Analysis of relative gene expression data using real-time quantitative PCR and the $2-\Delta \Delta C T$ method. Methods 25: 402-408, 2001.

29. Wu Y, Zhang P, Dai Q, Yang X, Fu R, Jiang L and Fang B: Effect of mechanical stretch on the proliferation and differentiation of BMSCs from ovariectomized rats. Mol Cell Biochem 382: 273-282, 2013.

30. Yang Z, Huang JH, Liu SF, Zhao YJ, Shen ZY, Wang YJ and Bian Q: The osteoprotective effect of psoralen in ovariectomy-induced osteoporotic rats via stimulating the osteoblastic differentiation from bone mesenchymal stem cells. Menopause 19: 1156-1164, 2012.

31. Li CW, Liang B, Shi XL and Wang H: Opg/Rankl mRNA dynamic expression in the bone tissue of ovariectomized rats with osteoporosis. Genet Mol Res 14: 9215-9224, 2015.

32. Faienza MF, Ventura A, Marzano F and Cavallo L: Postmenopausal osteoporosis: the role of immune system cells. Clin Dev Immunol 2013: 575936, 2013.

33. Kararigas G, Nguyen BT and Jarry H: Estrogen modulates cardiac growth through an estrogen receptor $\alpha$-dependent mechanism in healthy ovariectomized mice. Mol Cell Endocrinol 382: 909-914, 2014

34. Gong J, Meng HB, Hua J, Song ZS, He ZG, Zhou B and Qian MP: The SDF-1/CXCR4 axis regulates migration of transplanted bone marrow mesenchymal stem cells towards the pancreas in rats with acute pancreatitis. Mol Med Rep $9: 1575-1582,2014$.

35. Wu Q, Shao H, Darwin ED, Li J, Li J, Yang B, Webster KA and Yu H: Extracellular calcium increases CXCR4 expression on bone marrow-derived cells and enhances pro-angiogenesis therapy. J Cell Mol Med 13: 3764-3773, 2009.
36. Bhakta S, Hong P and Koc O: The surface adhesion molecule CXCR4 stimulates mesenchymal stem cell migration to stromal cell-derived factor-1 in vitro but does not decrease apoptosis under serum deprivation. Cardiovasc Revasc Med 7: 19-24, 2006.

37. Wynn RF, Hart CA, Corradi-Perini C, O'Neill L, Evans CA, Wraith JE, Fairbairn LJ and Bellantuono I: A small proportion of mesenchymal stem cells strongly expresses functionally active CXCR4 receptor capable of promoting migration to bone marrow. Blood 104: 2643-2645, 2004.

38. Long MY, Li HH, Pen XZ, Huang MQ, Luo DY and Wang PS: Expression of chemokine receptor-4 in bone marrow mesenchymal stem cells on experimental rat abdominal aortic aneurysms and the migration of bone marrow mesenchymal stem cells with stromal-derived factor-1. Kaohsiung J Med Sci 30: 224-228, 2014.

39. Qian D, Gong J, He Z, Hua J, Lin S, Xu C, Meng H and Song Z: Bone marrow-derived mesenchymal stem cells repair necrotic pancreatic tissue and promote angiogenesis by secreting cellular growth factors involved in the SDF-1 $\alpha /$ CXCR 4 axis in rats. Stem Cells Int 2015: 302015, 2015.

40. Zou Z, Zhang Y, Hao L, Wang F, Liu D, Su Y and Sun H: More insight into mesenchymal stem cells and their effects inside the body. Expert Opin Biol Ther 10: 215-230, 2010.

41. Karp JM and Leng Teo GS: Mesenchymal stem cell homing: the devil is in the details. Cell Stem Cell 4: 206-216, 2009.

42. Honczarenko M, Le Y, Swierkowski M, Ghiran I, Glodek AM and Silberstein LE: Human bone marrow stromal cells express a distinct set of biologically functional chemokine receptors. Stem Cells 24: 1030-1041, 2006.

43. Safwani WK, Makpol S, Sathapan S and Chua KH: Alteration of gene expression levels during osteogenic induction of human adipose derived stem cells in long-term culture. Cell Tissue Bank 14: 289-301, 2013.

44. Choi MR, Kim HY, Park JY, Lee TY, Baik CS, Chai YG, Jung KH, Park KS, Roh W, Kim KS, et al: Selection of optimal passage of bone marrow-derived mesenchymal stem cells for stem cell therapy in patients with amyotrophic lateral sclerosis. Neurosci Lett 472: 94-98, 2010.

45. Zhao D, Li XP, Gao M, Zhao C, Wang JL and Wei LH: Stromal cell-derived factor $1 \alpha$ stimulates human endometrial carcinoma cell growth through the activation of both extracellular signal-regulated kinase 1/2 and Akt. Gynecol Oncol 103: 932-937, 2006.

46. Bobis-Wozowicz S, Miekus K, Wybieralska E, Jarocha D, Zawisz A, Madeja Z and Majka M: Genetically modified adipose tissue-derived mesenchymal stem cells overexpressing CXCR4 display increased motility, invasiveness, and homing to bone marrow of NOD/SCID mice. Exp Hematol 39: 686-696, 2011.

47. Brennecke P, Arlt MJ, Campanile C, Husmann K, Gvozdenovic A Apuzzo T, Thelen M, Born W and Fuchs B: CXCR4 antibody treatment suppresses metastatic spread to the lung of intratibial human osteosarcoma xenografts in mice. Clin Exp Metastasis 31: 339-349, 2014.

48. Yu J,LiM,QuZ, Yan D,LiD and Ruan Q: SDF-1/CXCR4-mediated migration of transplanted bone marrow stromal cells toward areas of heart myocardial infarction through activation of PI3K/Akt. J Cardiovasc Pharmacol 55: 496-505, 2010

49. Yu ZH, Wang YX, Song Y, Lu HZ, Hou LN, Cui YY and Chen HZ. Up-regulation of $\mathrm{KCa} 3.1$ promotes human airway smooth muscle cell phenotypic modulation. Pharmacol Res 77: 30-38, 2013.

50. Kollet O, Shivtiel S, Chen YQ, Suriawinata J, Thung SN, Dabeva MD, Kahn J, Spiegel A, Dar A, Samira S, et al: HGF, SDF-1, and MMP-9 are involved in stress-induced human CD34 ${ }^{+}$ stem cell recruitment to the liver. J Clin Invest 112: 160-169, 2003.

51. Shahzad U, Li G, Zhang Y, Li RK, Rao V and Yau TM: Transmyocardial revascularization enhances bone marrow stem cell engraftment in infarcted hearts through SCF-C-kit and SDF-1-CXCR4 signaling axes. Stem Cell Rev 11: 332-346, 2015.

52. Liu N, Tian J, Cheng J and Zhang J: Migration of CXCR4 gene-modified bone marrow-derived mesenchymal stem cells to the acute injured kidney. J Cell Biochem 114: 2677-2689, 2013.

This work is licensed under a Creative Commons

Attribution-NonCommercial-NoDerivatives 4.0 International (CC BY-NC-ND 4.0) License. 